\title{
Endovascular electroencephalography: the technique and its application during carotid amytal assessment
}

\author{
S J Boniface, N Antoun
}

Department of Clinical Neurophysiology

S J Boniface

Department of

Radiology,

Addenbrookes

NHS trust,

Cambridge, UK

N Antoun

Correspondence:

Dr Simon Boniface,

Department of Clinical

Neurophysiology,

Addenbrookes NHS Trust,

Hills Road, Cambridge

CB2 2QQ, UK.

Received 22 September 1995 and in final revised form 26 September 1996

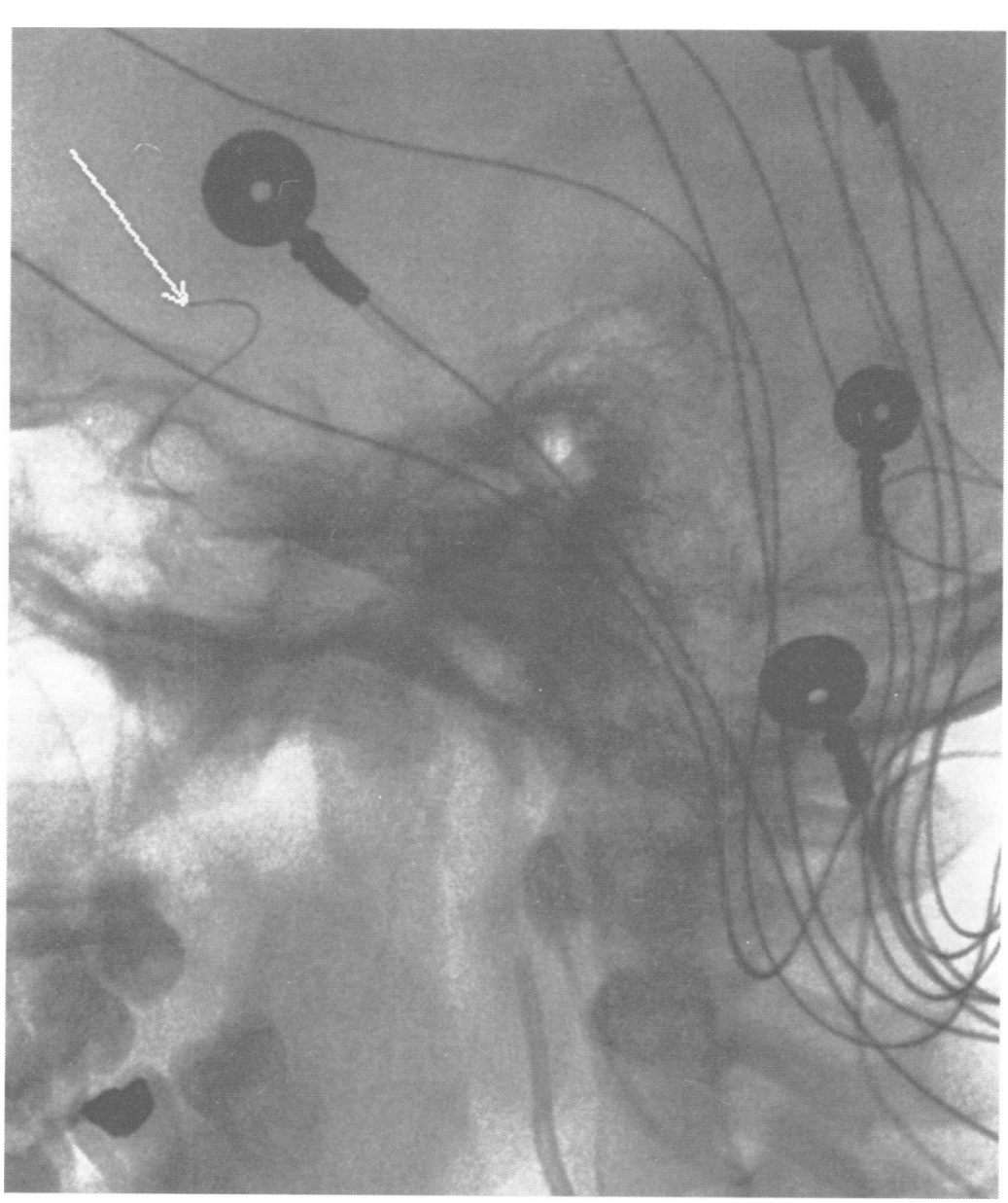

Figure 1 Plain radiograph showing the positions of the catheter in the external carotid artery and the intra-arterial electrode (arrow).

\begin{abstract}
The EEG was recorded using an endovascular guide wire as the electrode. This was Teflon coated except for its terminal portion, which was placed distal to the origin of the middle cerebral artery. Four hemispheres were sampled sequentially in two patients with intractable complex partial seizures who were undergoing routine preoperative carotid amytal assessment. Epileptiform spikes were detected in one patient, concordant with decreased hippocampal volume on MRI and scalp EEG changes. Endovascular EEG during the amytal procedure is less invasive than other intracranial EEG
\end{abstract}

\section{(a)} function with the carotid amytal procedure is often employed in those patients who are found to have temporal lobe lesions, ${ }^{2}$ a procedure known to influence the distribution of human hippocampal spikes. ${ }^{3}$ The aim of this study was to assess the feasibility of combining these approaches to obtain intracranial EEG using a minimally invasive method. ${ }^{4}$

\section{Methods}

Two men aged 20 and 34 years old with a protracted history of intractable complex partial seizures were studied. In patient 1 attacks involved an aura of thoughts racing through his mind, followed by movements of the right hand with lip smacking, sometimes evolving to tonic-clonic seizures starting in the right leg. In patient 2 , seizures started with a dusty smell or strange taste lasting a few seconds followed by loss of consciousness and sleepiness on recovery. Brain MRI in both patients had disclosed that the left hippocampus was diffusely smaller than the right.

Coaxial selective intracranial carotid artery catheterisation via the femoral approach under local anaesthetic and full heparinisation was performed with informed consent. The seeker (Standard 14 steerable guide wire; Target Therapeutics) was a tapered radio-opaque silicone sprayed stainless steel guide wire with an overall resistance and length of $33 \mathrm{ohm}$ and $175 \mathrm{~cm}$ respectively. Laminated polymer surface (Teflon) covered the distal $40 \mathrm{~mm}$ wire 
Figure 2 Ten second portion of left frontotemporal EEG from patient 1. The endovascular guide wire ( $G W$ ) channel is shown at the bottom. For clarity, EEG channels lacking any abnormalities have been omitted. (A) Bipolar montage; $(B)$ average reference montage. (Calibration bars $=1 \mathrm{~s}$ and $150 \mu \mathrm{V}$.)

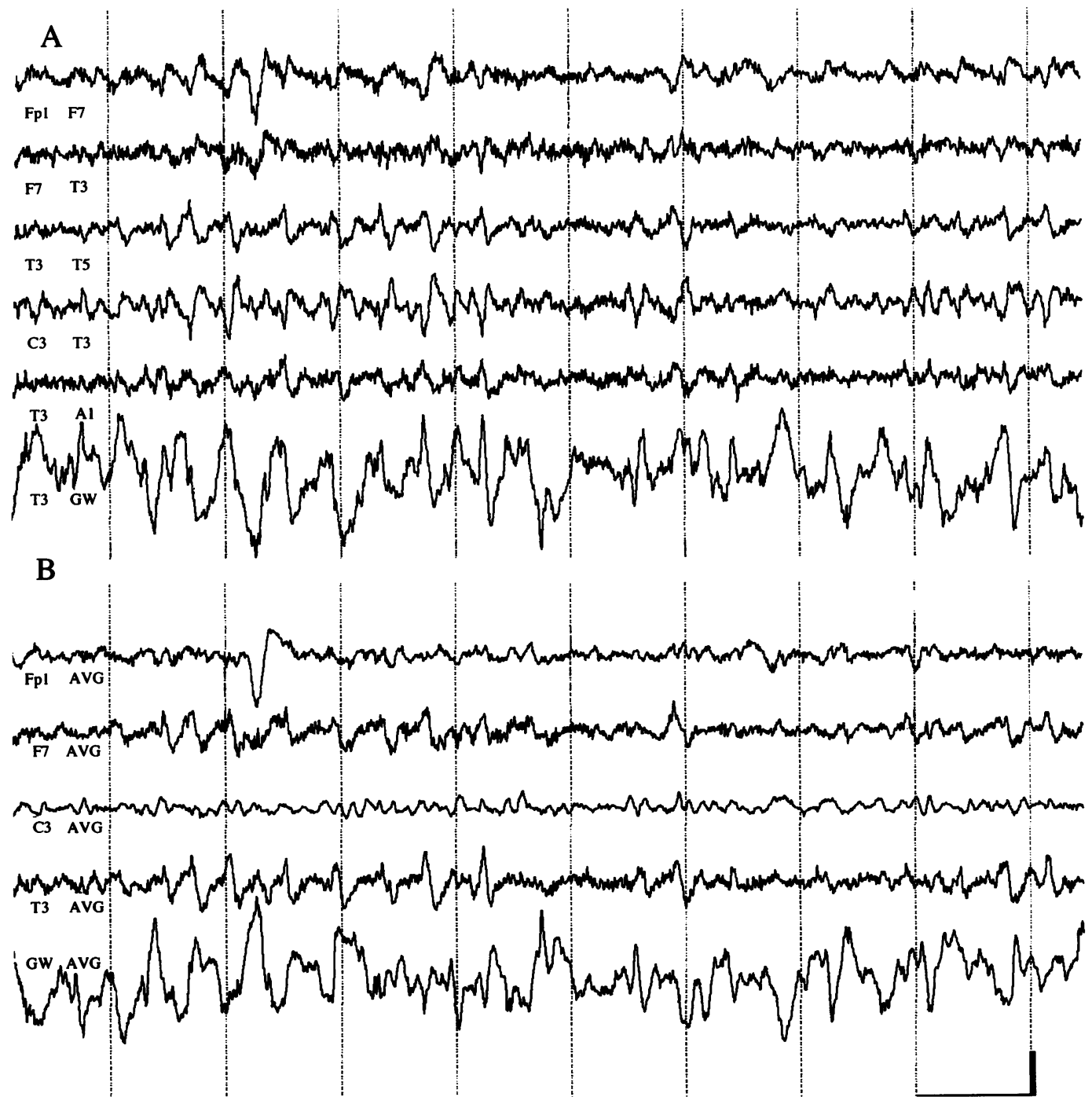

core but the distal most $20 \mathrm{~mm}$ were not covered by Teflon and made of a platinum coil.

The left hemisphere was studied first in both patients. The distal end of the catheter was positioned extracranially in the internal carotid artery and the guide wire was advanced to the origin of the middle cerebral artery (fig 1). Endovascular EEG recordings were obtained before, during, and after the injection of sodium amytal. Pulse artefact was a common problem. Simultaneous digital surface EEG recordings were obtained (Walter Graphtek) with 10-20 electrode placements and standardised neuropsychological assessments were performed.

\section{Results}

In patient 1 scalp EEG showed left midtemporal spikes and sharp waves (phase reversing at T3) throughout the procedure, which were temporarily reduced in frequency by the second of two left carotid injections. In patient 2, occasional left midtemporal focal sharp waves were detected. Ipsilateral slow activity swiftly followed the carotid injections in both patients with some spread to the contralateral hemisphere.
Endovascular EEG spikes were detected in patient 1 and reconstructed off line in bipolar and average reference formats (fig 2). Surface and endovascular EEG spikes were detected at a frequency of up to $1 / \mathrm{s}$ before carotid infection. In a typical run of 12 of these spikes reconstructed in average reference format, four were detected with greatest amplitude at the intra-arterial electrode, four at the $\mathrm{T} 3 \mathrm{elec}-$ trode, two at F7, and an additional two spikes with equal amplitudes at T3 and F7 (fig 2B)

\section{Discussion}

This study has shown that endovascular EEG is a feasible technique that can identify intracranial epileptiform abnormalities. It augmented the average reference format and has the potential to achieve more in a bipolar format when the electrical characteristics of the electrode are optimised. One advantage of this technique is the ability to move the guide wire between different intracranial sites. This ability may contribute to our understanding of the important distinction between volume conduction and neurophysiological propagation in the localisation of ictal and interictal onset, ${ }^{5}$ particularly if simultaneous recordings from multipolar devices or from both hemispheres could be achieved. 
Additional clinical applications await further study. The ability to reposition the guide wire in accordance with on line endovascular EEG findings may be of use for source localisation in the presurgical investigation of individual patients, with a lower expected complication rate than more invasive methods. It might also be of use during pharmacological activation with short acting barbiturates, a technique for which sphenoidal electrodes are currently used in some centres. The potential for the use of this technique in presurgical assessment, and possibly during endovascular theraputic procedures, is very encouraging.
We thank consultant neurologist Dr Stephen Wroe, and Miss Fiona Cameron and Mrs Julia Young for their technical and secretarial support respectively.

1 Polkey CE and Binnie CD. Assessment and selection of candidates for surgical treatment of epilepsy. Epilepsia 1995;36(suppl 1):41-5.

2 Rausch R, Silfvenius $\mathrm{H}$, Wiesser $\mathrm{HG}$, et al. Intra-arterial amobarbital procedures. In: Engel J Jr, ed. Surgical treatment of the epilepsies. New York: Raven Press, 1993. ment of
$341-58$.

3 Adachi N, Onuma T, Suzuki I, Shimizu H, Ishijima B. Intracarotid amobarbital injection produces hippocampal EEG changes in patients with temporal lobe epilepsy. EEG changes in patients with

Epilepsy Research 1993;15:75-8.
Boniface SJ, Antoun N. Intravascular EEG. Electroencephalogr Clin Neurophysiol 1996;98:4:42P.

5 Alarcon G, Guy CN, Binnie CD, Walter SR, Elwes RDC Polkey CE. Intracerebral propagation of interictal activity in partial epilepsy: implications for source localisation. $\mathcal{F}$ Neurol Neurosurg Psychiatry 1994;57:435-49. 